\title{
Messgenauigkeit von kalorimetrischen Massenstromsensoren
}

\author{
Peter Otto; Hans-Jürgen Postberg
}

Postberg+Co. GmbH, Emilienstraße 37, 34121 Kassel;

Tel.: +49561 506309-70, Fax: +49 561 506309-71

peter.otto@postberg.com; hj.postberg@postberg.com

\author{
Siegfried Hilbrich \\ Fakt GmbH Grüntenstr. 5, 87751 Heimertingen \\ Tel.: +49 8335 9888-0, Fax: +49 8335 9888-10 \\ shilbrich@fakt.com
}

\section{Einleitung}

Der physikalische Grundeffekt der modernen kalorimetrischen Massenstromsensoren beruht auf einem beheizten Sensorelement (Film, Keramik, Glas etc.), dessen elektrischer Widerstand von der Temperatur abhängt. Durch die Umströmung des Sensorelements im Medium findet ein lokaler Wärmetransport statt, welcher mit dem Massenstrom (kg/s) bzw. der Strömungsgeschwindigkeit korreliert (m/s). Bei der Messung kompressibler Medien, wie beispielsweise Luft, wird zur Umrechnung auf Nm/s die Mediumstemperatur zur Kompensation parallel gemessen. Eine Messung des Absolutdrucks zur Druckkompensation entfällt, da bei Druck > 1 bar $_{\mathrm{a}}$ der Massenstrom konstant bleibt.

Als Ausgangssignal der kalorimetrischen Massenstromsensoren ist in der Regel nicht der Massenstrom gewünscht, sondern der Normvolumenstrom $\left(\mathrm{Nm}^{3} / \mathrm{h}\right)$. Grundsätzlich geht für die Umrechnung in das Messsignal der Innendurchmesser bzw. die Querschnittfläche $\left(\mathrm{m}^{3}\right)$ der Messblöcke als Rechengröße mit ein. Durch falsche Innendurchmesserangaben entstehen Messfehler im zweistelligen Bereich. Die Prozessschnittstelle, d.h. die Schnittstelle zwischen Massenstromsensor und Rohrleitung spielt eine übergeordnete Rolle für die Grundgenauigkeit [1]. Ist diese Voraussetzung erfüllt, hat die kalorimetrische Massenstrommessung folgende Vorteile in der technischen Praxis gegenüber anderen physikalischen Messsystemen:

- Schnelle Reaktionsgeschwindigkeit auf Änderungen der Volumenströme, bzw. keine Trägheit durch bewegte Teile (vgl. Drehkolbenzähler)

- Wesentlich größere Messbereichsdynamik (vgl. Differenzdruck-Blendenmessungen)

- Kein statischer oder dynamischer Druckabfall, auch bei hohen Volumenströmen durch die Sensoren

- Hohe messtechnische Reproduzierbarkeit der Sensorik und Messarmaturen/ -blöcke

- Sichere und einfache Rekalibrierung des Messsensors

- Kostengünstiger in der Herstellung

\section{Aufgabenstellung}

Da kalorimetrische Massenstromsensoren kostenvorteile gegenüber den alternativen Messsystemen bieten, werden diese oft auch bezüglich der Messgenauigkeit in Frage gestellt, so bald diese mit „hochwertigen“ bzw. teueren Systemen verglichen werden. Dies ist zum Teil berechtigt, wenn das kalorimetrische Messsystem auf die Sensorik (Elektronik) reduziert und ohne Mechanik ausgeliefert wird. Um eine maximale Messgenauigkeit sicherzustellen, wird folgend die gesamte Messkette betrachtet. Diese beinhalten sowohl den kalorimetrischen Massenstromsenor als auch die Messtrecke inkl. Messblock oder Messarmatur.

Unter diesen Grundbedingungen wurde folgende Aufgabenstellung an das kalorimetrische Messsystem gestellt. Für die Praxisanwendung im Bereich der Prüfstände wurde eine Messgenauigkeit von <=1\% Messfehler auf den Messwert gefordert. Da ein kalorimetrischer Massenstromsensor aus elektronischen Bauteilen mit Fertigungsschwankungen hergestellt wird, setzt dies voraus, dass ein Kalibrier- und Zertifizierungsprüfstand verfügbar ist, der eine bessere Grundgenauigkeit hat. Hinzu kommt ein zu lösender physikalischer Nebeneffekt, dass die messtechnische Praxis der Prüfstände im Überdruckbereich arbeiten. Obwohl kalorimetrische Massenstromsensoren nahezu druckunabhängig sind, kommen durch Kalibrierung unter Atmosphäre Messfehler von >2\% hinzu. D.h. der kalorimetrische 
Sensor muss unter Druck kalibriert und zertifiziert werden können, wenn dieser unter Druck in der Praxis eingesetzt werden soll.

\section{Anwendung im Prüfstandsbau}

Für thermomechanische und thermodynamische Versuche in der Automobilindustrie werden moderne

Heißgassimulatoren eingesetzt. Die Simulatoren ermöglichen typische Anwendungen abzubilden, wie:

- Temperaturwechselprüfungen

- Temperaturschockprüfungen

- Strömungsuntersuchungen

- Ermittlung von Leistung $n$ und Wirkungsgraden

- Überprüfung der thermomechanischen Festigkeit

- Untersuchungen von thermodynamischen Parametern

Eckdaten der Versuche:

- Temperaturen im Heisgasstrom bis $1300^{\circ} \mathrm{C}$

- Massendurchsatz von wenigen $\mathrm{kg} / \mathrm{h}$ bis mehrere tausend Kilogramm pro Stunde

- Temperaturgradient von mehreren hundert Kelvin pro Sekunde

- Drücke bis 8.000 mbarü

- Genauigkeit <1\% vom Messwert

- Wiederholbarkeit $<0,2 \%$

Für Heißgassimulationsanlagen werden höchste Anforderungen an die Mess- und Regeltechnik gestellt. Herzstück solcher Anlagen sind Heißgaserzeuger wie HG200 oder HG400 der FAKT GmbH, welche explizit für solche Aufgaben entwickelt wurden (HG200) bis $200 \mathrm{~kW}$ und seit Jahren im Prüflabor der FAKT Gmbh sowie bei System- und Komponentenherstellern oder Forschungslaboren im Einsatz sind.

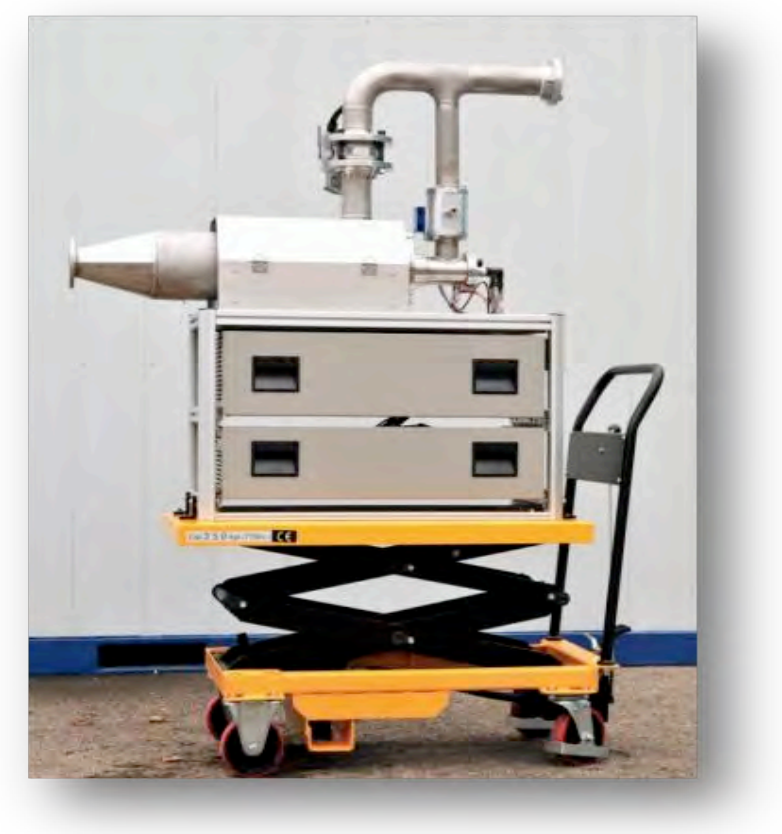

Abbildung 1: Heißgaserzeuger HG200 mit Messtechnik (Quelle: FAKT GmbH)

Der Heißgaserzeuger soll Druck, Temperatur und Luftmasse in einem weiten Bereich parametrierbar halten und die Betriebspunkte verschiedenster Motoren nachbilden. Dazu ist eine exakte Regelung von Prozessluft und Gas-Luft-Gemisch nötig. Mitentscheidend für das Gesamtsystem ist also die Qualität der Messtechnik zur Erfassung der Gase. Diese muss einen möglichst weiten Messbereich abdecken, über schnelle Ansprechzeiten (Dynamik), hohe Messgenauigkeit und mechanische Reproduzierbarkeit auch im Rekalibrierungsfall verfügen, eine kompakte Bauform/ Geometrie haben und zudem betriebssicher und wartungsfreundlich sein [1].

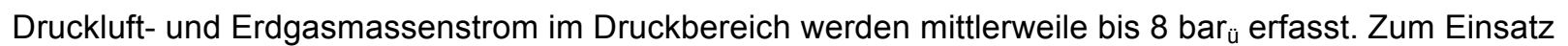
für die Massenstrommessung Druckluft kommen kalorimetrische Sensoren auf Basis von 
Dünnschichtelementen aus Glas, da Sie über eine Messbereichsspanne von 1:400 verfügen, keinen Druckverlust erzeugen und große Messdynamik ( $\mathrm{t}_{90}<100 \mathrm{~ms}$ ) haben.

\section{Physikalische und messtechnische Möglichkeiten}

Messklassen sind eindeutig definierte Genauigkeitsangaben kompletter Messwertketten. Angaben über die Genauigkeit der Sensoren allein sagen noch nichts über den Messfehler der gesamten Messkette aus. Während die Leistungen der kalorimetrischen Massenstromsensoren, Auswerteelektroniken und Korrekturrechner genau beschrieben und geprüft werden, gibt es für ihren mechanischen Einbau so gut wie keine verbindlichen Vorschriften [2].. Ein Sensor oder ein Korrekturrechner, der unter Prüfbedingungen mit einer Genauigkeit von $0,3 \%$ misst, sagt nichts über die reproduzierbare Genauigkeit in der Praxis aus

\begin{tabular}{|llll|}
\hline Messklasse & Klasse 0,5 & Klasse 1 & Klasse 2 \\
Bezeichnung & Referenzklasse & Abrechnungsausführung & Industrieausführung \\
\hline Messfehler & $2 \%$ & $5 \%$ & $\pm 10 \%$ \\
\hline Anwendung & Kalibrierprüfstände & Kontrollmessung & Industrielle Messung \\
\hline Prüftstand & ÖKD oder DKD Prüfstand & Nach ISO 6358 & Kein Prüfstand \\
\hline
\end{tabular}

Tabelle 1: Messklassen [1]

Um die Messklasse 0,5 für die gesamte Messwertkette zu erreichen ist das Zusammenspiel von Einzelkompenenten notwendig:

- Mechanischer Prozessschnittstelle für den Einbau

- Neuster Kalorimetrischer Sensoren mit Korrekturrechner inkl. Druckkompensation

- ÖKD oder DKD Kalibrier- und Zertifizierungsprüfstand

\subsection{Kalorimetrische Sensoren}

Kritisch ist die Sicherstellung der kundenseitig geforderten Messgenauigkeit von unter $1 \%$ auf dem Messwert, da bisherige Kalibriermöglichkeiten als Referenzprüfstände unter Druck fehlten. Hinzu kommen erste Messungen und Untersuchungen aus dem Jahr 2008 auf dem Volumenstromprüfstand der Hochschule Amberg-Weiden, welche eine geringe aber tendenzielle Abhängigkeit der Messgenauigkeit vom Druck in DN25 ergaben, obwohl diese Massenstromsensoren bisher als druckunabhängig galten.

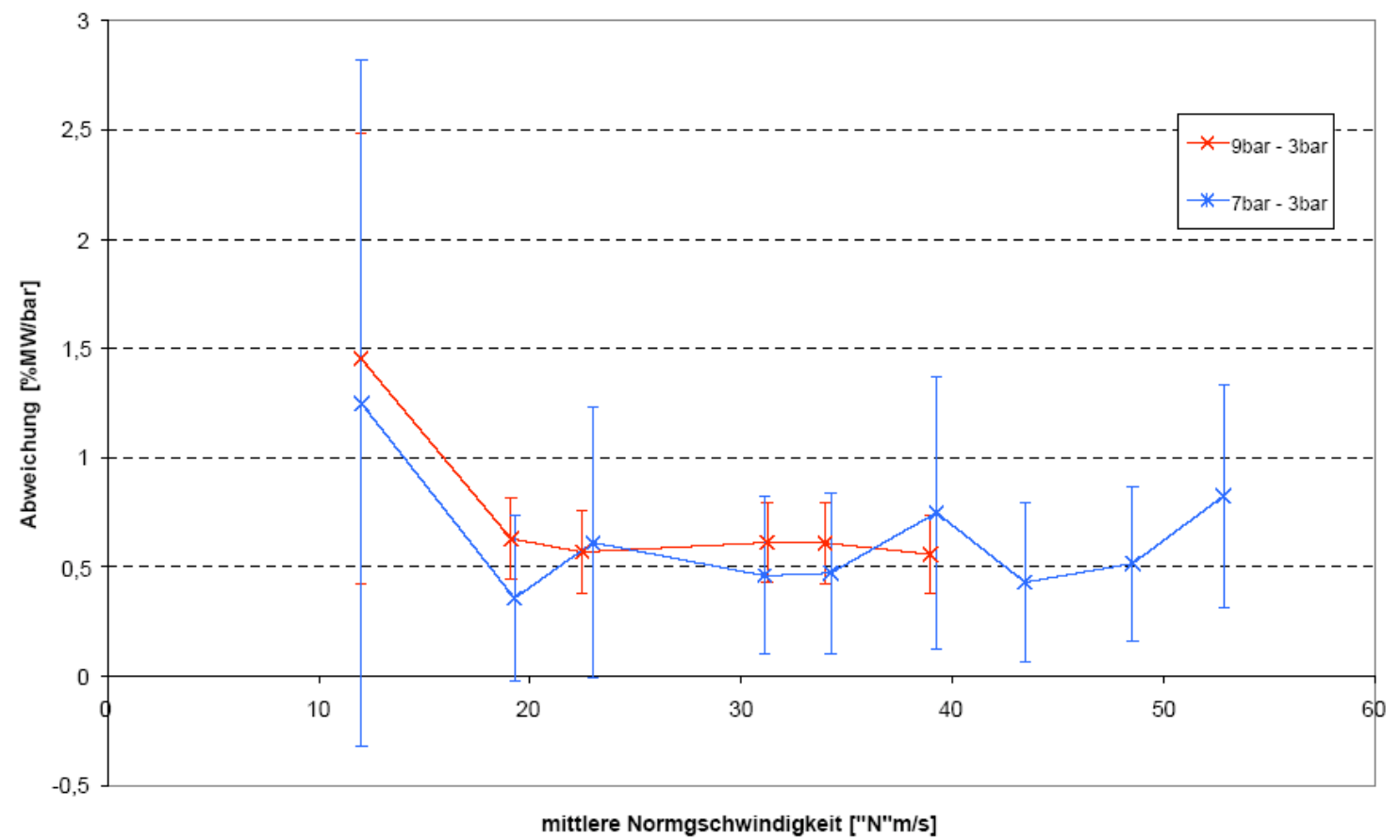

Abbildung 2: Erster Beleg der Druckabhängigkeit des Massenstromsensors (Quelle: Postberg+Co. GmbH, 2008) 
Bei variierenden Drücken im Bereich 4 - 8 bar $_{\ddot{u}}$ bei den Simulatoren entspricht dies einem max. Fehler von 3\% auf den Messwert. Bei geforderten Messgenauigkeit von 1\% ist diese Abweichung nicht mehr tolerierbar und bedarf der messtechnischen Korrektur. Diese Erkenntnis ist auch für Messungen bis 40 bar $_{\ddot{u}}$ Druckluft von messtechnischer Bedeutung. Die Erklärung für diese Druckabhängigkeit thermischer Sensoren wurde theoretisch und praktisch belegt [3].

Die neuentwickelten Korrekturrechner der Firma Postberg+Co. GmbH wurden entsprechend dieser Erkenntnis seit 2008 weiterentwickelt. Die Korrekturrechner BSx, EEx und die PB+COntroller verfügen über einen 4-20 mA Druckeingang, der mittels Korrekturformel bei schwankenden Drücken diesen automatisch korrigiert. Somit wird die Messgenauigkeit für Systeme mit großen Druckschwankungen (z.B. PET Flaschenherstellung) sichergestellt. Der Fehler zwischen Auslegungsdrücken von 40 bar $_{\ddot{u}}$ und

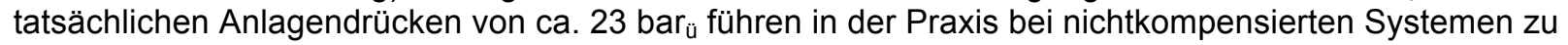
zweistelligen Messfehlern

\subsection{Mechanische Prozessschnittstelle}

Auch die hohen Massenströme von bis zu $1.500 \mathrm{~kg} / \mathrm{h}$ (ca. $1.200 \mathrm{Nm}^{3} / \mathrm{h}$ ) Druckluft für kompakte Bauformen von (DN32 und DN50) führen zu besonderen Anforderungen an die Prozessschnittstelle (Mechanik). Diese kommen bei Prüfständen der Firma FAKT, bei falsch ausgelegten Druckluftnetzen bzw. aber 40-bar Druckluftnetzen, in der Praxis vor.

Für die Sicherstellung der Messgenauigkeit des kalorimetrischen Sensors bedarf es der reproduzierbaren Messung in der Mitte, 100\% zylindrischen Messblockgeometrien und glatte Flanschübergänge mittels messtechnisch optimierten $\mathrm{PB}+\mathrm{COmpac} 囚-$ Flanschen mit O-Ring Dichtungen. Erste Voruntersuchungen der Firma E+E Elektronik ergaben bei Randmessungen eine unzureichende Reproduzierbarkeit der Messergebnisse und dadurch einen zu großen Messfehler. Durch intensive Zusammenarbeit der Firmen Posterg+Co. und des Sensorikunternehmens E+E Elektronik ist es gelungen, diese Optimierungspotentiale konsequent zu nutzen und bei den aktuell befindlichen Messarmaturen und Messblöcken zu vertreiben.
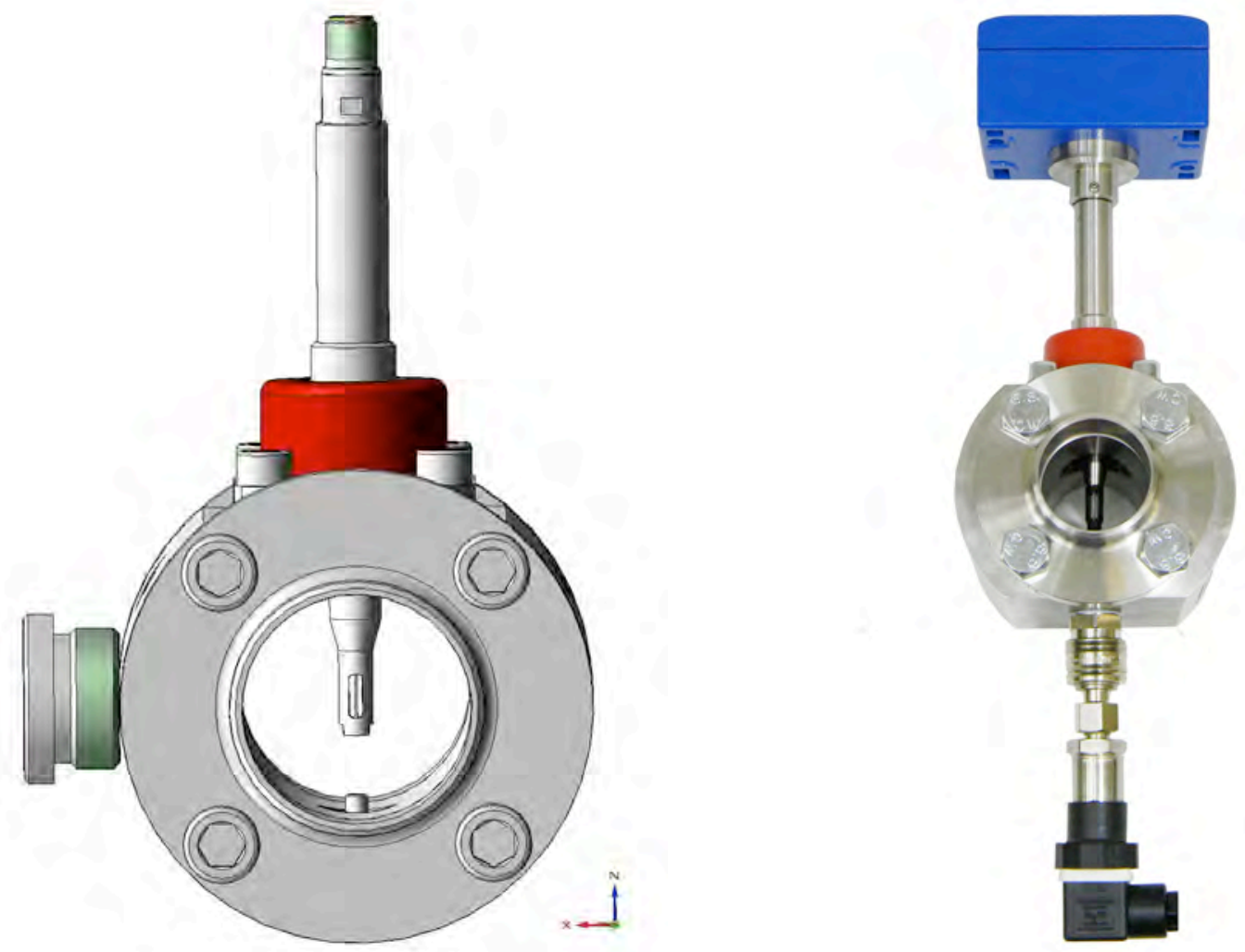

Abbildung 3: 3D-CAD Modell des Messblocks mit PB+COmpac ${ }^{\circledR}$ Vorschweißflansche inkl. kalorimetrischen Massenstromsensor (links); Umsetzung als basic001s Druckluftzähler mit Druckkompensation (rechts) 
Die Prozessschnittstelle muss zusätzlich sicherstellen, dass bei Reinigung oder jährlicher Rekalibrierung bzw. Rezertifizierungen der Sensorik der Ein- und Ausbau des Sensors repoduzierbar ist. Geringe Abweichungen der Eintauchtiefe und Ausrichtung (Geometrie) führt systembedingt zu Messfehlern, da thermische Massenstrommessungen die Punktmessung durchführen.

4.3 Österreichischer Kalibrierdienst für Volumenstrom (ÖKD Kalibrationslaboratorium 23)

Der neue ÖKD Prüfstand ermöglicht es bei Drücken von 0,1 bis $10 \mathrm{Mpa}\left(23^{\circ} \mathrm{C}+-3^{\circ} \mathrm{C}\right)$ Volumenströme von 0,6 bis $2.300 \mathrm{Nm}^{3} / \mathrm{h}$ mit einer Messgenauigkeit von $0,003 \mathrm{~m}^{3} / \mathrm{h}+0,9 \% \mathrm{f} . \mathrm{mv}$ zu dokumentieren. Dieser akkreditierte Prüfstand stellt eine rückführbare Messgenauigkeit sicher und ist in Europa einmalig in den genannten Spezifikationen.

\begin{tabular}{|c|c|c|c|c|c|}
\hline Position & $\begin{array}{c}\text { Referenz } \\
\text { Volumenstrom bei } \\
\text { Normbedingungen }\end{array}$ & Messwert & $\begin{array}{c}\text { Messwert } \\
\text { Volumenstrom bei } \\
\text { Normbedingungen }\end{array}$ & $\begin{array}{l}\text { Abweichung von } \\
\text { Referenzwert }\end{array}$ & $\begin{array}{l}\text { Erweiterte } \\
\text { Unsicherheit } \\
\text { der Prüfung }\end{array}$ \\
\hline Position & $\begin{array}{c}\text { Reference } \\
\text { flow at standard } \\
\text { conditions }\end{array}$ & $\begin{array}{l}\text { Output } \\
\text { value }\end{array}$ & $\begin{array}{c}\text { Output value } \\
\text { flow at standard } \\
\text { conditions }\end{array}$ & $\begin{array}{l}\text { Deviation from } \\
\text { reference value }\end{array}$ & $\begin{array}{c}\text { Extended } \\
\text { measurement } \\
\text { uncertainty }\end{array}$ \\
\hline \# & $\mathrm{m}^{3} / \mathrm{h}$ & $\mathrm{mA}$ & $\mathrm{m}^{3} / \mathrm{h}$ & $\mathrm{m}^{3} / \mathrm{h}$ & $\mathrm{m}^{3} / \mathrm{h}$ \\
\hline 1 & 29.9 & 4.312 & 29.3 & -0.6 & 0.3 \\
\hline 2 & 176.4 & 5.887 & 176.9 & 0.5 & 2.8 \\
\hline 3 & 502.8 & 9.387 & 505.1 & 2.3 & 7.1 \\
\hline 4 & 846.2 & 13.083 & 851.6 & 5.4 & 7.7 \\
\hline 5 & 1359.3 & 18.537 & 1362.8 & 3.5 & 14.9 \\
\hline
\end{tabular}

Normbedingungen von Luft:

Standard conditions of air

$\rho_{0}=1,29167 \mathrm{~kg} / \mathrm{m}^{3}$

Druck / pressure

Temperatur / temperature

Rel.Feuchte / rel. humidity

$$
\begin{aligned}
& \mathrm{p}_{0}=1013.25 \mathrm{hPa} \\
& \mathrm{T}_{0}=273.15 \mathrm{~K} \\
& \mathrm{U}_{\mathrm{w}}=45 \% \mathrm{rh}
\end{aligned}
$$

Tabelle 2: Messergebisse eines kalorimetrischen Massenstromsensors mit Druckkorrektur (ÖKD Zertifikat KA002008) inkl. Rahmenbedingungen inkl. erweiterte Unsicherheit der Prüfung [4].

Die angegebene erweiterte Messunsicherheit $U$ entspricht der zweifachen Standardunsicherheit $(k=2)$, welche für eine Normalverteilung einen Grad des Vertrauens von etwa 95\% bedeutet. Die Standardunsicherheit wurde in Übereinstimmung mit dem Leitfaden zur Angabe der Unsicherheit beim Messen (Engl. Original: „Guide to the Expression of Uncertainty in Measurement (BIPM, IEC, IFCC, ISO, IUAPC, IUPAP, OIML)") und damit gemäß Dokument EA-4/02 ermittelt.

Die Messwerte und die Abweichungen wurden aus dem Gesamtfluss der Referenz und einer Mittelwertbildung von mindestens 10 Messwerten des Prüflings in einem Zeitraum von zumindest 1 min berechnet.

Der Österreichische Kalibrierdienst nimmt neben den Sensor- und Korrekturrechner-ID-Nummern (eindeutige Identifikationsnummer) auch die ID-Nummer der Prozessschnittstelle (Messblock $\mathrm{PB}+\mathrm{COmpac}\left({ }^{\circledR}\right)$ mit auf, da diese wie bereits beschrieben direkten Einfluss auf die Messgenauigkeit der Messwertkette hat. Auch der während der Kalibrierung aktive Kalibrierdruck in der Rohrleitung am Prüfling wird mit dokumentiert. Dieser kann nach Vorgabe während der ÖKD Zertifizierung im angegebenen Druckbereich schwanken.

\section{Zusammenfassung}

In den Jahren 2011/2012 ist es gelungen durch die Entwicklung eines druckkompensierten kalorimetrischen Massenstromsensors, einer neuen mechanischen Prozessschnittstelle und einem neuen Prüfstand des Österreichischen Kalibrierdienst (ÖKD) alle erforderlichen Voraussetzungen zu schaffen, um technisch/ wissenschaftlich:

- die Druckabhängigkeit der Messgenauigkeit von kalorimetrischen Sensoren,

- die Reproduzierbarkeit von CNC gefertigten Messblöcken mit PB+COmpac®-Flansch

- die unzureichende Kalibrierung von atmosphärischen Prüfständen $>50 \mathrm{Nm} / \mathrm{s}$ 
zu dokumentieren. Für den beschriebenen Prüfstand der Firma FAKT ist somit die optimale Voraussetzung geschaffen, die Anforderungen seiner Kunden sicherzustellen.

Die geforderte Messgenauigkeit wurde auf die gesamte Messkette ausgeweitet und dokumentiert. Diese beinhaltet auch den mechanischen Einbau, welcher als Prozessschnittstelle die Genauigkeit in der Praxis sicherstellt. Die Messergebnisse des ÖKD dokumentieren, dass eine Messklasse 0,5 erreichbar ist. Für moderne Prüfstände, wie im Fall der Firma FAKT, wurde somit ein neuer Stand der Technik erreicht.

\section{Referenzen:}

[1] Stehle, Peter; Otto, Peter; Postberg, Hans-Jürgen, VDI-Bericht 1681 (2002), „Volumenstrommessung schafft Transparenz"

[2] Otto, Peter; Postberg, Hans-Jürgen, Maschinenmarkt (6/2008), „Geschickter Einsatz von Messtechnik stellt Druckluftverfügbarkeit sicher"

[3] Rohm, Matthias, (2009) „Uncertainty estimation in the measurement of standardized air flow in industrial applications using thermal flow sensor"

[4] Österreichischer Kalibrierdienst; Linder, Ewald, ÖKD 232008 (2012) „Durchflussmessgerät für Luft, BS73/PB+COmpac DN 50“ 